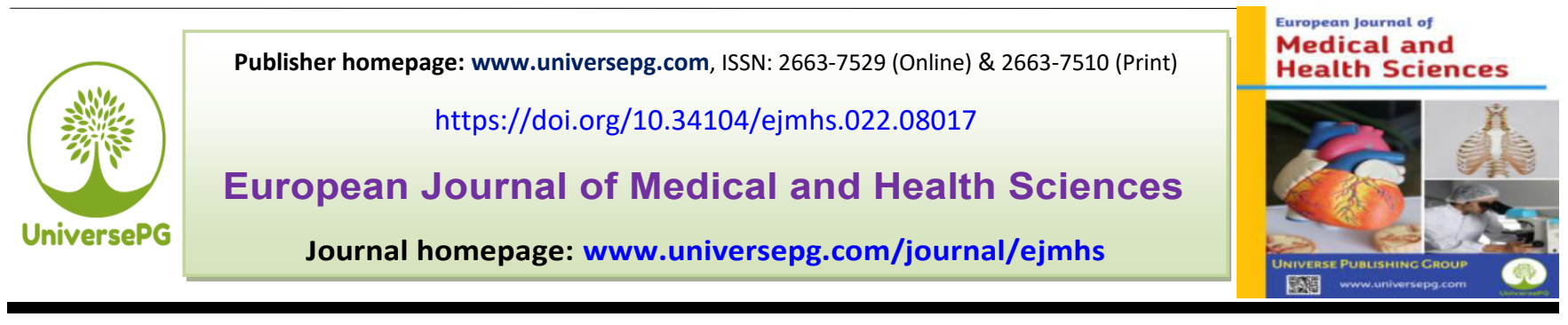

\title{
Effect of a Ketogenic Diet on Body Weight and Lipid Profile
}

\author{
Nabeela Kanwal ${ }^{1}$, Seemin Kashif ${ }^{2}$, Muhammad Kashif ${ }^{3}$, Allaudin ${ }^{4}$, and Gulalai Rehman ${ }^{5 *}$ \\ ${ }^{1}$ Dept. of Dermatology, PIMS hospital G8/3 Islamabad, Pakistan; ${ }^{2}$ Dept. of Environmental Design \& Nutritional Sciences, \\ Allama Iqbal Open University, Islamabad, Pakistan; ${ }^{3}$ Dept. of Anesthesia, Prince Sultan Military Medical City, Riyadh, \\ Kingdom of Saudi Arabia; ${ }^{4}$ Dept. of Surgery, Bolan Medical College Quetta and Fellow Bariatric Surgery Shifa International \\ Hospital, Islamabad, Pakistan; and ${ }^{5}$ Dept. of Nutrition, Baluchistan Institute of Nephro-Urology, Quetta, Pakistan. \\ *Correspondence: drgulalai@yahoo.com (Dr. Gulalai Rehman, Consultant Nutritionist, Department of Nutrition, Baluchistan \\ Institute of Nephro-Urology, Quetta, Pakistan).
}

\begin{abstract}
This review article aimed to study the effects of a ketogenic diet on obesity-related measures, and lipid profiles. Total 22 articles were included in the review. Anthropometric measures included in the review were total body mass (Wt), fat mass (FM), body mass index (BMI), and waist circumference (WC). Lipid profile included in this review was serum cholesterol level, serum HDL, serum LDL, and serum triglycerides (TAG). The shortest intervention in these studies lasted for 4 days, and the longest intervention lasted for 12 months. There was a significant Wt loss in all studies. Significant reduction in BMI, FM, and WC was also observed in many studies. In most studies, variable effects on lipid profile were observed. Serum TC level fell in 10 studies and serum TAG levels decreased in 13 studies, but this reduction was statistically insignificant in most studies. Serum LDL level fell in 10 studies and increased in 3 studies, and serum HDL levels fell in 7 studies and increased in 6 studies, but these changes were also not statistically significant in most studies. This review article found beneficial effects of ketogenic diet weight loss and lipid profile, but the studies in this review included dietary intervention of a period $\leq 12$ months. Studies for extended periods (at least $\geq 2$ years) should be done to observe long-term effects of a ketogenic diet.
\end{abstract}

Keywords: Ketogenic diet, high-fat diet, BMI, weight loss, HDL, lipid profile, Type2 DM, and NAFLD.

\section{INTRODUCTION:}

Ketogenic diet $(\mathrm{KD})$ is a diet characterized by a very low carbohydrate content i.e. $5 \%-10 \%$ of total daily intake, or 20-50 g per day (Watanabe et al., 2020). A very-low-calorie ketogenic diet (VLCKD) plan is divided into phases. The first phase is with a calorie intake of 600-800 kcal/day and restricted carbohydrates, resulting in a ketosis state. This state is pursued until 80$90 \%$ of the normal weight is obtained. The second phase includes the progressive introduction of carbohydrates. Calorie intake is increased from 800 to 1500 $\mathrm{kcal} /$ day until the normal weight is achieved. The last maintenance phase is with an intake of 1500-2000 UniversePG I www.universepg.com $\mathrm{kcal} / \mathrm{day}$. The second and third phases are not ketogenic (Perticone et al., 2019). Rapid weight loss associated with KD makes it one of the most popular diets. Along with being a tough diet due to strict carbohydrate restrictions, it is also an expensive diet as cheaper carbohydrates are exchanged by expensive meat, low-carb flours, and specific types of fats like MCT, butter, and nuts. Adverse health consequences of a ketogenic diet are believed to be due to its high saturated fat content. Some researchers have reported lipid profile derangements following this diet (Batch et al., 2020; Muscogiuri et al., 2019), which is expected to happen following a very high-fat diet. But contrary 
to this expectation, many studies reported an improvement in lipid profile (Batch et al., 2020; Min-istrini et al., 2019; Valenzano et al., 2019).

This review article was aimed at studying outcomes of KD and VLCKD on obesity-related measures, and on lipid profile. It also discussed the effects of KD on certain obesity-related diseases.

\section{MATERIALS AND METHODS:}

\section{Review of Literature}

A systematic literature search Google scholar was performed to identify various studies published since 2017. Only those studies were included which were done on "human subjects", with overweight or obesity. Studies were included which evaluated the effects of KD and VLCKD on blood sugar control, decrease in fat mass, and lipid profile as main measures. The search terms were composed of a combination of keywords "ketogenic diet or high-fat diet", "weight loss", "lipid profile", "NAFLD", and "Type2 DM". Reference lists of review articles were also screened to identify eligible studies. A total of 49 articles were extracted. Two articles were removed because they were animal studies. Other 17 articles were removed because 12 of them were review articles, one was an editorial, 3 were opinions, and one was a case report. Three more articles were removed because they included only one gender. Further 5 articles were removed because they did not contain desired outcome variables. Hence total 22 articles were selected (Fig. 1).

\section{Inclusive/exclusive criteria}

Inclusive criteria were: (1) studies including ketogenic $\operatorname{diet}(\mathrm{KD})$ or very-low-calorie ketogenic diet (VLCKD), (2) studies including obese patients, with or without any chronic disease, and (3) studies carried out on humans. Exclusive criteria were: (1) case report studies, (2) meta-analysis or review studies, (3) editorials and opinions, (4) studies on animals, (5) studies on one gender only, and (6) studies not having desired outcome variables.

\section{Outcome measures}

Outcome measures included anthropometric measures and biochemical measures. Anthropometric measures included in the review were total body mass (weight= $\mathrm{W})$, fat mass (FM), body mass index (BMI), and waist circumference (WC). Biochemical measures included lipid profile i.e. serum total cholesterol (TC), serum high-density lipoprotein (HDL), and serum low-density lipoprotein (LDL), and serum triglycerides (TAG).

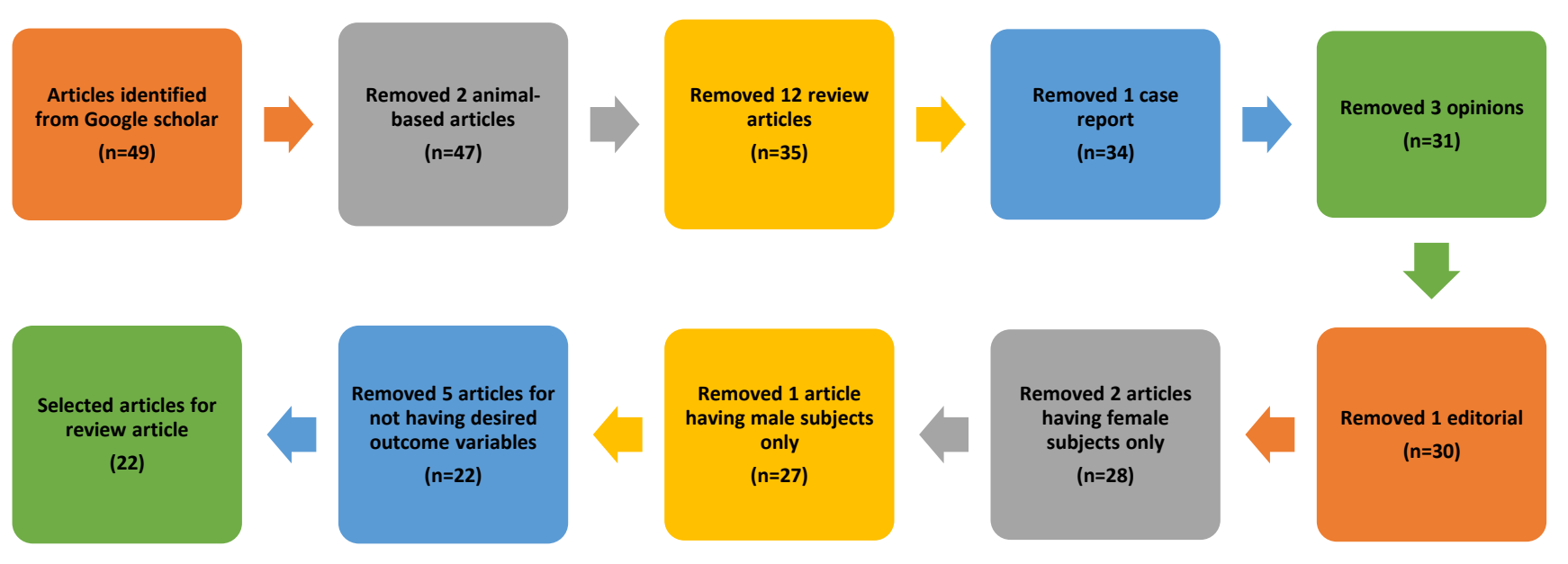

Fig. 1: Study selection flow-diagram.

\section{RESULTS:}

In our review, the dietary intervention lasted for $\leq 5$ weeks in 10 studies, and for $>5$ weeks in 12 studies. The shortest intervention in these studies lasted for 4 days, and the longest intervention lasted for 12 months (Table 1).

\section{Anthropometric measures}

A significant change in $\mathrm{Wt}$ was observed in most studies in the review (Table 1). This decline in $\mathrm{Wt}$ actually reflected total fat loss, which included loss of visceral fat also. The studies which did not mention body weight, reflected fat loss in form of change in 
BMI, fat mass (FM), or waist circumference (WC). Out of total 22 studies, 18 studies showed a significant reduction in body weight (total body mass). Similarly, there was a significant loss of fat in 12 studies, a significant reduction in BMI in 13 studies, and a significant reduction in WC in 7 studies. Only 2 studies were of shorter than a week duration (Luukkonen et al., 2020), still a significant decrease in $\mathrm{Wt}$ and other anthropometric measures was observed.

\section{Biochemical measures}

KD affected lipid profile variably. In most studies, the effects were present but were not statistically significant. Serum TC level fell in 10 studies, though this reduction was not statistically significant in many of these studies. Similarly, serum LDL level fell in 10 studies, however, it was found to increase in 3 studies, and serum HDL levels fell in 7 studies and increased in 6 studies. Shifts in HDL, LDL, and TAG levels were also not statistically significant in most studies. The influence of length of interventions was also overviewed. Only 2 studies were shorter than a week duration (Luukkonen et al., 2020; Myette-Côté et al.,
2018), still significant decrease in $\mathrm{Wt}$ and other anthropometric measures was observed. In one of these studies (Luukkonen et al., 2020), insignificant fall in TC, HDL, and LDL levels, but in the other study (Myette-Côté et al., 2018), insignificant rise in serum triglyceride levels was observed. Significant decrease in weight and/or other anthropometric measures in all other studies of 2-5 weeks duration were observed. In 3 of these studies, a decline in lipids was observed which was significant. The remaining studies showed an insignificant decrease in lipid levels. Two studies had insignificant increase in HDL level. Like the short duration studies, longer duration studies $(=>5$ weeks) also showed significant decline in weight and/or all anthropometric measures. Out of 12 studies, 8 studies included lipid profile. TC level fell statistically significantly in 4 out of 8 studies and insignificantly in one study, and TAG level fell significantly in 6 out of 8 studies, and insignificantly in one of these studies. In a study, HDL level fell significantly, and in other two studies fell insignificantly.

Table 1: Characteristics of trials of ketogenic diet published in last five years and their effects on obesity and lipid profile.

\begin{tabular}{|c|c|c|c|c|c|c|c|c|}
\hline $\begin{array}{c}\text { First } \\
\text { Author, } \\
\text { year }\end{array}$ & Country & $\begin{array}{l}\text { Study } \\
\text { design }\end{array}$ & $\begin{array}{l}\text { Participants of } \\
\text { intervention/ } \\
\text { Control groups }\end{array}$ & Intervention & $\begin{array}{c}\text { Duration of } \\
\text { ketogenic } \\
\text { Phase (weeks) }\end{array}$ & Inclusion criteria & $\begin{array}{c}\text { Study } \\
\text { objective }\end{array}$ & $\begin{array}{c}\text { Parameters with } \\
\text { effects \& } \\
\text { Significance }\end{array}$ \\
\hline $\begin{array}{l}\text { Myette- } \\
\text { Côté, } \\
2018\end{array}$ & Canada & $\begin{array}{c}\text { Randomized } \\
\text { crossover } \\
\text { study }\end{array}$ & $11 / 11$ & $\begin{array}{c}\text { Low-carb, } \\
\text { high-fat diet }\end{array}$ & 4 days & $\begin{array}{c}\text { Adults with } \\
\text { Type } 2 \text { DM, } \\
\text { age } 40-75 \text { years, } \\
\text { both genders }\end{array}$ & $\begin{array}{c}\text { Effect of low- } \\
\text { carbohydrate, } \\
\text { high-fat diet } \\
\text { with/without } \\
\text { post-meal } \\
\text { walks on } \\
\text { type2 DM } \\
\text { patients }\end{array}$ & $\begin{array}{c}\mathbf{W t} \downarrow^{\mathbf{1}} \\
T A G^{2}\end{array}$ \\
\hline $\begin{array}{c}\text { Luukkonen, } \\
2020\end{array}$ & Finland & $\begin{array}{c}\text { Nutritional } \\
\text { interventiona } \\
1 \text { study }\end{array}$ & 10/None & $\mathrm{KD}$ & 6 days & $\begin{array}{c}\text { Over } \\
\text { weight/ } \\
\text { obese adults, } \\
\text { both genders }\end{array}$ & $\begin{array}{l}\text { KD reverses } \\
\text { NAFLD and } \\
\text { insulin } \\
\text { resistance } \\
\text { despite } \\
\text { increasing } \\
\text { circulating } \\
\text { non-esterified } \\
\text { fatty acids } \\
\text { (NEFA) }\end{array}$ & $\begin{array}{c}\mathbf{W t} \downarrow \\
\mathbf{F M} \downarrow \\
\mathbf{B M I} \downarrow \\
\mathbf{W C} \downarrow \\
\\
T C \downarrow \\
H D L \downarrow \\
L D L \downarrow\end{array}$ \\
\hline $\begin{array}{l}\text { Choi, } \\
2018\end{array}$ & Korea & $\begin{array}{c}\text { Randomized } \\
\text { controlled } \\
\text { trial }\end{array}$ & $19 / 11$ & KD & 2 weeks & $\begin{array}{c}\text { Age } 19-49 \text { years, } \\
\text { BMI>25, } \\
\text { both genders }\end{array}$ & $\begin{array}{l}\text { Effects of } \\
\text { low-calorie } \\
\text { ketogenic } \\
\text { nutrition } \\
\text { drinks }\end{array}$ & $\begin{array}{c}\mathbf{W t} \downarrow \\
\mathbf{F M} \downarrow \\
\mathbf{B M I} \downarrow \\
\mathbf{W C} \downarrow \\
T C \downarrow \\
H D L \downarrow \\
L D L \downarrow \\
T A G \downarrow\end{array}$ \\
\hline
\end{tabular}




\begin{tabular}{|c|c|c|c|c|c|c|c|c|}
\hline $\begin{array}{c}\text { Merra, } \\
2017\end{array}$ & Italy & $\begin{array}{c}\text { Crossover } \\
\text { randomized } \\
\text { double-blind } \\
\text { trial }\end{array}$ & $54 / 54$ & VLCKD & 3 weeks & $\begin{array}{l}18-65 \text { years, } \\
\text { BMI } \geq 25, \\
\text { both genders }\end{array}$ & $\begin{array}{l}\text { VLCKD and } \\
\text { KD used as } \\
\text { part of } \\
\text { lifestyle } \\
\text { modification }\end{array}$ & $\begin{array}{c}\mathbf{F M} \downarrow \\
\mathbf{B M I} \downarrow \\
\mathbf{W C} \downarrow \\
T C \downarrow \\
H D L \downarrow \\
L D L \downarrow \\
T A G \downarrow \\
\end{array}$ \\
\hline $\begin{array}{c}\text { Buscemi et } \\
\text { al., } 2021\end{array}$ & Italy & Cohort & $31 / 20$ & VLCKD & $10-12$ weeks & $\begin{array}{c}\text { Both genders, } \\
\text { 18-65 years, } \\
\text { BMI } 27-39.9 \text {, } \\
\text { and non- } \\
\text { diabetic or type } \\
\text { 2DM from }<6 \\
\text { years with } \\
\text { HbA1c } \leq 10 \%\end{array}$ & $\begin{array}{c}\text { VLCKD } \\
\text { and vitamin } \\
\text { D levels. }\end{array}$ & $\begin{array}{c}\mathbf{W t} \downarrow \\
\mathbf{F M} \downarrow \\
\mathbf{B M I} \downarrow \\
\mathbf{W C} \downarrow \\
\mathrm{TC} \downarrow \\
\mathrm{HDL} \downarrow \\
\mathrm{TAG} \downarrow\end{array}$ \\
\hline $\begin{array}{c}\text { Gomez } \\
\text { Arbelaez, } \\
2018\end{array}$ & Spain & $\begin{array}{c}\text { Nutritional } \\
\text { interventiona } \\
1 \text { study }\end{array}$ & 20/None & VLCKD & 4 months & $\begin{array}{c}\text { Age } 18-65 \\
\text { years, } \\
\text { BMI } \geq 30, \\
\text { both genders }\end{array}$ & \begin{tabular}{|} 
Changes in \\
RMR, and the \\
associated \\
hormonal \\
alterations in \\
patients with a \\
VLCK-diet
\end{tabular} & $\begin{array}{r}\mathbf{W t} \downarrow \\
\mathbf{B M I} \downarrow\end{array}$ \\
\hline $\begin{array}{c}\text { Sajoux, } \\
2019\end{array}$ & Spain & Cohort & $20 / 59$ & VLCDK & $2-3$ moths & $\begin{array}{l}\text { Overweight/ob } \\
\text { ese adults, } \\
\text { both genders }\end{array}$ & \begin{tabular}{|c|} 
VLCK diet \\
can change \\
body \\
composition
\end{tabular} & $\begin{array}{l}\mathbf{W t} \downarrow \\
\mathbf{F M} \downarrow\end{array}$ \\
\hline $\begin{array}{c}\text { Bruci, } \\
2020\end{array}$ & Italy & $\begin{array}{c}\text { Observationa } \\
1 \text { prospective } \\
\text { study }\end{array}$ & 92/None & VLCKD & 3 months & $\begin{array}{c}\text { Obese patients } \\
\text { coming to High } \\
\text { Specialization } \\
\text { Centre for the } \\
\text { Care of Obesity, } \\
\text { Sapienza Uni- } \\
\text { versity of Rome, } \\
\text { both genders }\end{array}$ & $\begin{array}{c}\text { Efficacy of } \\
\text { VLCKD in } \\
\text { mild } \\
\text { kidney } \\
\text { failure. }\end{array}$ & $\begin{array}{r}\mathbf{W t} \downarrow \\
\mathbf{F M} \downarrow \\
\mathbf{B M I} \downarrow \\
\\
\mathbf{T C} \downarrow \\
H D L \downarrow^{2} \\
L D L \downarrow \\
\text { TAG } \downarrow\end{array}$ \\
\hline
\end{tabular}

\begin{tabular}{|c|c|c|c|c|c|c|c|c|}
\hline $\begin{array}{c}\text { Pilone, } \\
2018\end{array}$ & Italy & \begin{tabular}{|c|} 
Nutritional \\
interventional \\
study
\end{tabular} & 119/None & VLCKD & 30 days & $\begin{array}{l}\text { Age } 18-62 \text { years, } \\
\text { BMI } \geq 40, \\
\text { both genders }\end{array}$ & $\begin{array}{l}\text { Evaluation } \\
\text { of effect of } \\
\text { VLCKD }\end{array}$ & $\begin{array}{c}\mathbf{W t} \downarrow \downarrow^{\mathbf{1}} \\
\mathbf{F M} \downarrow \\
\mathbf{B M I} \downarrow \\
\mathbf{W C} \downarrow \\
T C \downarrow^{2} \\
H D L \uparrow \\
L D L \downarrow \\
T A G \downarrow\end{array}$ \\
\hline $\begin{array}{c}\text { Valenzano, } \\
2019\end{array}$ & Italy & \begin{tabular}{|c|} 
Nutritional \\
interventional \\
study
\end{tabular} & 20/None & VLCKD & 8 weeks & $\begin{array}{l}\text { Obese adults, } \\
\text { age } 20-60 \text { years, } \\
\text { both genders }\end{array}$ & $\begin{array}{l}\text { Effect of } \\
\text { VLCKD }\end{array}$ & $\begin{array}{c}\mathbf{W t} \downarrow \\
\mathbf{F M} \downarrow \\
\mathbf{B M I} \downarrow \\
\mathbf{T C} \downarrow \\
H D L \downarrow \\
\mathbf{L D L} \downarrow \\
\mathbf{T A G} \downarrow\end{array}$ \\
\hline $\begin{array}{c}\text { McKenzie, } \\
2017\end{array}$ & USA & $\begin{array}{c}\text { Nutritional } \\
\text { interventio } \\
\text { nal study }\end{array}$ & $238 /$ None & VLCKD & 10 weeks & $\begin{array}{l}\text { Type } 2 \mathrm{DM}, \\
\text { age } 21-65 \text { years, } \\
\text { both genders }\end{array}$ & $\begin{array}{l}\text { Individuals } \\
\text { with T2D } \\
\text { could } \\
\text { achieve } \\
\text { weight loss. }\end{array}$ & $\begin{array}{c}\mathbf{W t} \downarrow \\
\mathbf{B M I} \downarrow \\
\mathbf{T C} \downarrow \\
H D L \uparrow \\
L D L \uparrow \\
\mathbf{T A G} \downarrow\end{array}$ \\
\hline
\end{tabular}




\begin{tabular}{|c|c|c|c|c|c|c|c|c|}
\hline $\begin{array}{c}\text { Volek, } \\
2019\end{array}$ & USA & $\begin{array}{c}\text { Nutritional } \\
\text { interventio } \\
\text { nal study }\end{array}$ & $14 / 15$ & KD & 12 weeks & $\begin{array}{c}\text { Healthy adults } \\
\text { from various } \\
\text { military branches, } \\
\text { both genders }\end{array}$ & $\begin{array}{c}\text { Metabolic, } \\
\text { performance } \\
\text { responses of } \\
\text { KD }\end{array}$ & $\begin{array}{c}\mathbf{W t} \downarrow \\
\mathbf{F M} \downarrow\end{array}$ \\
\hline
\end{tabular}

\begin{tabular}{|c|c|c|c|c|c|c|c|c|}
\hline $\begin{array}{c}\text { Walton, } \\
2019\end{array}$ & USA & \begin{tabular}{|c|} 
Nutritional \\
interventional \\
study
\end{tabular} & 11/None & $\begin{array}{l}\text { Low-carb, } \\
\text { high-fat } \\
\text { diet }\end{array}$ & 90 days & $\begin{array}{c}\text { Age } 18-45 \text { years, } \\
\text { females, } \\
\text { type } 2 \mathrm{DM}\end{array}$ & $\begin{array}{c}\text { Low-carbohydrate } \\
\text { diets are effective } \\
\text { to improve } \\
\text { insulin resistance }\end{array}$ & $\begin{array}{l}\mathbf{W t} \downarrow^{\mathbf{1}} \\
\mathbf{B M I} \downarrow \\
\mathbf{H D L} \uparrow \\
L D L \downarrow^{2} \\
\text { TAG } \downarrow\end{array}$ \\
\hline Castro, 2018 & Spain & $\begin{array}{c}\text { Nutritional } \\
\text { interventional } \\
\text { study }\end{array}$ & 20/None & VLCDK & 4 months & $\begin{array}{c}\text { Age } 18-65 \text { years } \\
\text { BMI } \geq 30, \\
\text { both genders }\end{array}$ & Weight loss & $\mathbf{F M} \downarrow$ \\
\hline $\begin{array}{c}\text { Greene, } \\
2018\end{array}$ & Australia & $\begin{array}{l}\text { Randomized, } \\
\text { crossover } \\
\text { design }\end{array}$ & $12 / 12$ & LCKD & 6 months & $\begin{array}{c}14 \text { power lifters } \\
\text { and weight } \\
\text { lifters, } \\
\text { both genders, } \\
\text { age range } 18-55 \\
\text { years }\end{array}$ & $\begin{array}{l}\text { LCKD could } \\
\text { be used by } \\
\text { weightlifters }\end{array}$ & $\mathbf{W t} \downarrow$ \\
\hline $\begin{array}{l}\text { Perticone, } \\
2019\end{array}$ & Italy & \begin{tabular}{|c|} 
Nutritional \\
interventional \\
study
\end{tabular} & $28 / 28$ & VLCKD & 12 months & $\begin{array}{l}\text { Overweight/o } \\
\text { bese adults, } \\
\text { both genders }\end{array}$ & $\begin{array}{l}\text { Ketogenic diet } \\
\text { effect on } \\
\text { vitamin D } \\
\text { levels }\end{array}$ & $\begin{array}{c}\mathbf{W C} \downarrow \\
T C \downarrow \\
H D L \uparrow \\
L D L \downarrow \\
\mathbf{T A G} \downarrow\end{array}$ \\
\hline $\begin{array}{l}\text { Saslow, } \\
2017\end{array}$ & USA & $\begin{array}{c}\text { Randomized } \\
\text { controlled trial }\end{array}$ & $16 / 18$ & VLCKD & 12 months & $\begin{array}{c}\text { Aged } \geq 18 \\
\text { years, } \\
\text { BMI } \geq 25, \\
\text { HbA1c }>6.0 \%\end{array}$ & $\begin{array}{l}\text { Compare LCK } \\
\text { and moderate } \\
\text { carbohydrate } \\
\text { diet. }\end{array}$ & $\begin{array}{l}\mathbf{W t} \downarrow \\
\mathbf{B M I} \downarrow \\
H D L \uparrow \\
L D L \uparrow \\
T A G \downarrow\end{array}$ \\
\hline $\begin{array}{l}\text { significant } \\
\text { VLCKD }=\mathrm{v} \\
\text { serum total } \mathrm{c}\end{array}$ & low & $\begin{array}{l}\text { old letters, } \\
\text { orie ketogenic } \\
\text { HDL = serum }\end{array}$ &. & . & 1 & $\begin{array}{l}\text { dy mass index } \\
\text { y lipoprotein, } \mathrm{T}\end{array}$ & $\mathrm{VC}=$ waist circu & s \\
\hline
\end{tabular}

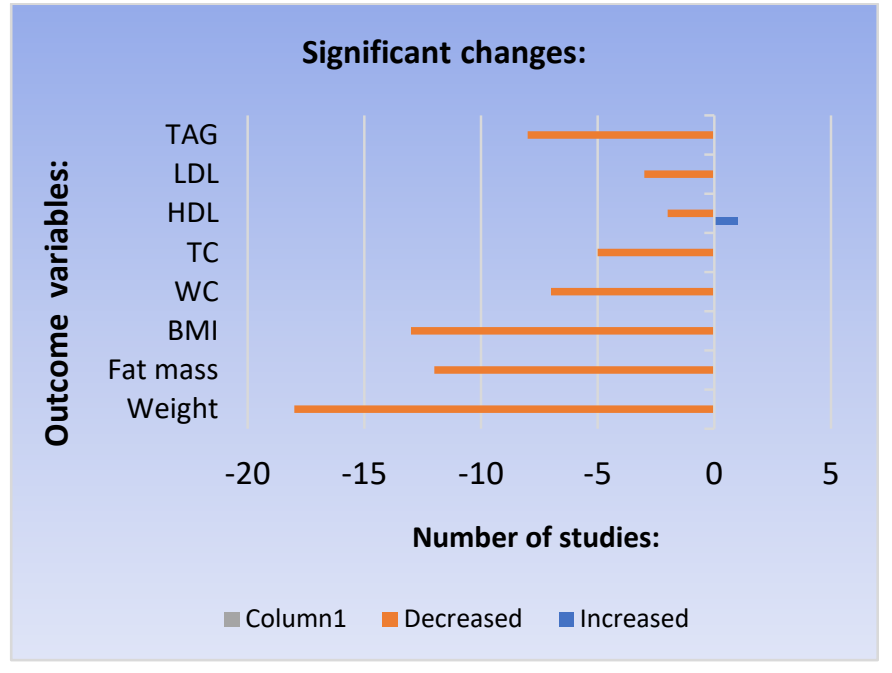

(a)

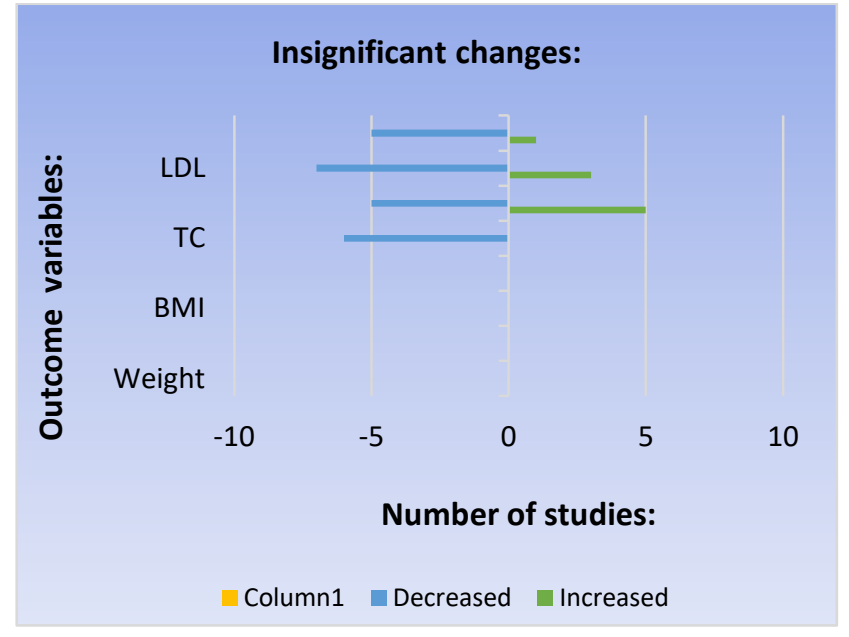

(b)

Fig. 2: Significant and insignificant changes after ketogenic diet. 


\section{Continued Table 1 -}

\begin{tabular}{|c|c|c|c|c|c|c|c|c|}
\hline $\begin{array}{l}\text { First } \\
\text { Author, } \\
\text { year }\end{array}$ & Country & $\begin{array}{l}\text { Study } \\
\text { design }\end{array}$ & $\begin{array}{l}\text { Participants of } \\
\text { intervention/ } \\
\text { Control groups }\end{array}$ & Intervention & \begin{tabular}{|c|}
$\begin{array}{c}\text { Duration of } \\
\text { ketogenic } \\
\text { Phase (weeks) }\end{array}$ \\
\end{tabular} & $\begin{array}{l}\text { Inclusion } \\
\text { criteria }\end{array}$ & $\begin{array}{c}\text { Study } \\
\text { objective }\end{array}$ & $\begin{array}{c}\text { Parameters } \\
\text { with effects \& } \\
\text { Significance }\end{array}$ \\
\hline $\begin{array}{c}\text { Albanese, } \\
2019\end{array}$ & Italy & \begin{tabular}{|c|} 
Nutritional \\
interventional \\
study
\end{tabular} & $72 / 106$ & VLCKD & 3 weeks & $\begin{array}{l}\text { Patients with } \\
\text { morbid obesity } \\
\text { undergoing lap- } \\
\text { aroscopic sleeve } \\
\text { gastrectomy, } \\
\text { both genders }\end{array}$ & $\begin{array}{l}\text { Weight loss in } \\
\text { candidates } \\
\text { und }\end{array}$ & $\mathbf{W t} \downarrow^{1}$ \\
\hline $\begin{array}{l}\text { Ministrini, } \\
2019\end{array}$ & Italy & $\begin{array}{c}\text { Nutritional } \\
\text { interventional } \\
\text { study }\end{array}$ & $52 / 20$ & VLCKD & 25 days & $\begin{array}{c}18-65 \text { years age, } \\
\text { both genders, } \\
\text { BMI } \geq 40 \text { or a } \\
\text { BMI } \geq 35 \text { with } \\
\text { comorbidities } \\
\text { (type } 2 \text { DM, HTN, } \\
\text { obstructive sleep } \\
\text { apnea etc) }\end{array}$ & $\begin{array}{l}\text { Low activity } \\
\text { of lysosomal } \\
\text { acid lipase } \\
\text { (LAL) could } \\
\text { be involved in } \\
\text { NAFLD }\end{array}$ & $\begin{array}{r}\mathbf{W t} \downarrow \\
\text { FM } \downarrow \\
\text { BMI } \downarrow \\
\text { LDL } \downarrow \\
\text { TAG } \downarrow\end{array}$ \\
\hline $\begin{array}{l}\text { D'Abbond- } \\
\text { anza, } 2020\end{array}$ & Italy & $\begin{array}{c}\text { Nutritional } \\
\text { interventional } \\
\text { study }\end{array}$ & $70 /$ None & VLCKD & 25 days & \begin{tabular}{|} 
Age $18-65$ years, \\
Both genders, \\
BMI $\geq 40$ or \\
BMI $\geq 35$ with \\
obesity-related \\
comorbidities. \\
\end{tabular} & \begin{tabular}{|c} 
Effects of sex \\
differences on \\
weight loss and \\
NAFLD in obese \\
patients
\end{tabular} & $\begin{array}{l}\mathbf{F M} \downarrow \\
\mathbf{W C} \downarrow\end{array}$ \\
\hline $\begin{array}{l}\text { Hall, } \\
2020\end{array}$ & USA & $\begin{array}{c}\text { Randomized, } \\
\text { crossover } \\
\text { design }\end{array}$ & $20 / 20$ & $\mathrm{KD}$ & 28 days & $\begin{array}{l}\text { Overweight/ob } \\
\text { ese adults, } \\
\text { both genders }\end{array}$ & $\begin{array}{l}\text { Compare low- } \\
\text { carbohydrate } \\
\text { diet with low- } \\
\text { fat diet }\end{array}$ & $\begin{array}{r}\mathbf{W t} \downarrow \\
T C \downarrow^{2} \\
\mathbf{H D L} \downarrow \\
L D L \uparrow \\
T A G \downarrow\end{array}$ \\
\hline $\begin{array}{l}\text { Schiavo, } \\
2018\end{array}$ & Italy & $\begin{array}{c}\text { Nutritional } \\
\text { interventio } \\
\text { nal study }\end{array}$ & 27/None & $\begin{array}{c}\text { Ketogenic } \\
\text { micronutrie } \\
\text { nt-enriched } \\
\text { diet }\end{array}$ & 4 weeks & $\begin{array}{l}\text { Morbidly obese } \\
\text { patients } \\
\text { undergoing } \\
\text { bariatric } \\
\text { surgery, } \\
\text { both genders }\end{array}$ & $\begin{array}{l}\text { Effect of } \\
\text { micronutrient- } \\
\text { rich KD in } \\
\text { reducing body } \\
\text { weight }\end{array}$ & $\begin{array}{c}\mathbf{W t} \downarrow \\
\mathbf{B M I} \downarrow \\
\mathbf{T C} \downarrow \\
H D L \uparrow \\
\mathbf{L D L} \downarrow \\
\text { TAG } \downarrow\end{array}$ \\
\hline
\end{tabular}

Similarly, HDL level increased significantly in a study, and insignificantly in 3 studies. Significant rise in HDL level occurred in 90 days intervention (Walton et al., 2019), and two insignificant increases in HDL level occurred in 12 months interventions (Perticone et al., 2019; Saslow et al., 2017). LDL levels fell significantly in one study (Valenzano et al., 2019), and insignificantly in 3 studies, but increased insignificantly in only 2 studies. There was no significant increase in LDL levels in any study.

\section{DISCUSSION:}

All studies constituting this review revealed a statistically significant reduction in body fat in terms of weight, fat mass, BMI, and WC. Our review also found correction of lipid profile in most studies. In this review, a variable effect of ketogenic diet was observed on lipid UniversePG I www.universepg.com profile. It was expected that a very high-fat diet would lead to derangements in the lipid profile. However, contrary to this expectation, a shift of lipid levels was found towards the healthy side. Serum TC declined in all the studies in which it was measured. Serum LDL and TAG levels increased insignificantly in some studies but fell in majority of the studies, but HDL levels increased in about $50 \%$ of these. Our pre-sent review, however, found a decrease in LDL levels in most studies, and even at 12 months of dietary intervention (Perticone et al., 2019).

\section{Effecton fat loss}

According to literature, ketogenic diet caused double the reduction of $\mathrm{Wt}$ on the 15th day of the intervention as compared to a diet with restricted calories only (Drabińska et al., 2021). This could be related to the 
differences in calorie intake. In experimental groups, the top reduction of body weight was recorded after six months, but the weight loss slowed down after twelve months, regardless of the diet followed. The diets were effective even after two years (Albanese et al., 2019; Batch et al., 2020; Drabińska et al., 2021). VLCKD also showed achievement of a significant decline in body weight in patients. Results were obtained during the ketogenic phase and weight remained stable over the next two years (Castellana et al., 2020). Our review of literature revealed that there was significant weight loss even in dietary intervention as short as four days.

\section{Effect on serum lipids}

In literature, the ketogenic diet showed a rise in HDL levels, along with a rise in amounts of LDL, and verylow-density lipoproteins (VLDL) in blood (Batch et al., 2020). However, in the present review, a variable effect of ketogenic diet was observed on lipid profile. A shift of lipid levels was found towards the healthy side. Serum TC levels declined in all studies, serum LDL and TAG levels increased insignificantly in some studies, but fell in most, and HDL levels increased in about half of them. Review found a decrease in LDL levels in most studies, even at 12 months (Perticone et al., 2019).

\section{Effect on NAFLD}

There is a common belief that increasing dietary fat intake leads to fatty liver and prevents fat mass loss. But a normo-caloric high-fat ketogenic diet (HFKD) has been found to inhibit lipogenesis and induce fatty acid oxidation, leading to weight loss and reduced heaptic fat. Conversely, a hypercaloric but proper diet diminishes fat oxidation in liver and increases lipogenesis from carbohydrates, causing NAFLD (Wata-nabe et al., 2020). At very low caloric intake, macro-nutrient disposition is not important in improving liver pathology. It is rather caused by weight loss itself. Whereas at higher calories, the macronutrient ratio seems to become more important. Carbohydrate restriction plays a primary role in the efficacy of KD on NAFLD. Ketosis might affect NAFLD pathogenesis, apart from carbohydrate restriction. Ketone esters reduced glycolysis and increased muscle fat oxidation in the absence of a carbohydrate-restricted diet when administered to athletes (Watanabe et al., 2020). Our review found improvement in lipid profile in most studies, and even a reduction in liver size and statuses in NAFLD patients (Pilone et al., 2018).

\section{Effect on Type2 DM}

Studies found that ketogenic diet could reduce fasting blood glucose and glycosylated hemoglobin, along with improving lipid metabolism and reducing body weight (Yuan et al., 2020). It also improved weight and metabolic parameters in Type2 DM patients (Choi et al., 2018; Di Raimondo et al., 2021). Although the ketogenic diet showed a favorable effect on HDL levels, there was an accompanying increase in amounts of LDL and very-low-density lipoproteins in the literature. Hence, it is recommended that those wishing to start a ketogenic diet, especially those with comorbidities like type $2 \mathrm{DM}$, and liver disease should consult their physicians for risks and benefits (Blanco et al., 2019; Di Raimondo et al., 2021; Gupta et al., 2017).

\section{Effect on cardiovascular diseases}

Obesity, hypertension, type 2 DM, and NAFLD are all connected, and all are major risk factors for cardiovascular diseases (Watanabe et al., 2020). KD results in decreased bodyweight, TAG, and diastolic blood pressure, and an increment in HDL levels as well (Gupta et al., 2017). All these changes are beneficial for the cardiovascular system.

\section{Effect on polycystic ovary syndrome (PCOS)}

PCOS is accompanied by obesity, insulin resistance, LH/FSH ratio, and androgen excess. KD helped treat PCOS by improving body weight, testosterone levels, luteinizing hormone/ follicle-stimulating hormone ratio, and insulin levels (Gupta et al., 2017). KD is not free of adverse events, if not carried out with proper care (Watanabe et al., 2020). If present in large amounts, $\beta$ hydroxybutyrate, and acetoacetate can result in a decreased blood $\mathrm{pH}$ (acidemia). A third ketone, acetone, is neutral, which does not alter blood $\mathrm{pH}$. Acetone can pass through lungs into one's breath, and can make it a ketone biomarker (Blanco et al., 2019).One case report narrated that KD offers short-term weight loss, but it can have potentially harmful side effects like ketoacidosis (Blanco et al., 2019). But this diet can be considered safe for weight loss in diseases like DM, because the ketosis during this diet does not go beyond 7 to $8 \mathrm{mM} / \mathrm{L}$, and hence the possibility of ketoacidosis in these patients is limited (Fig. 2) (Drabińska et al., 2021). 


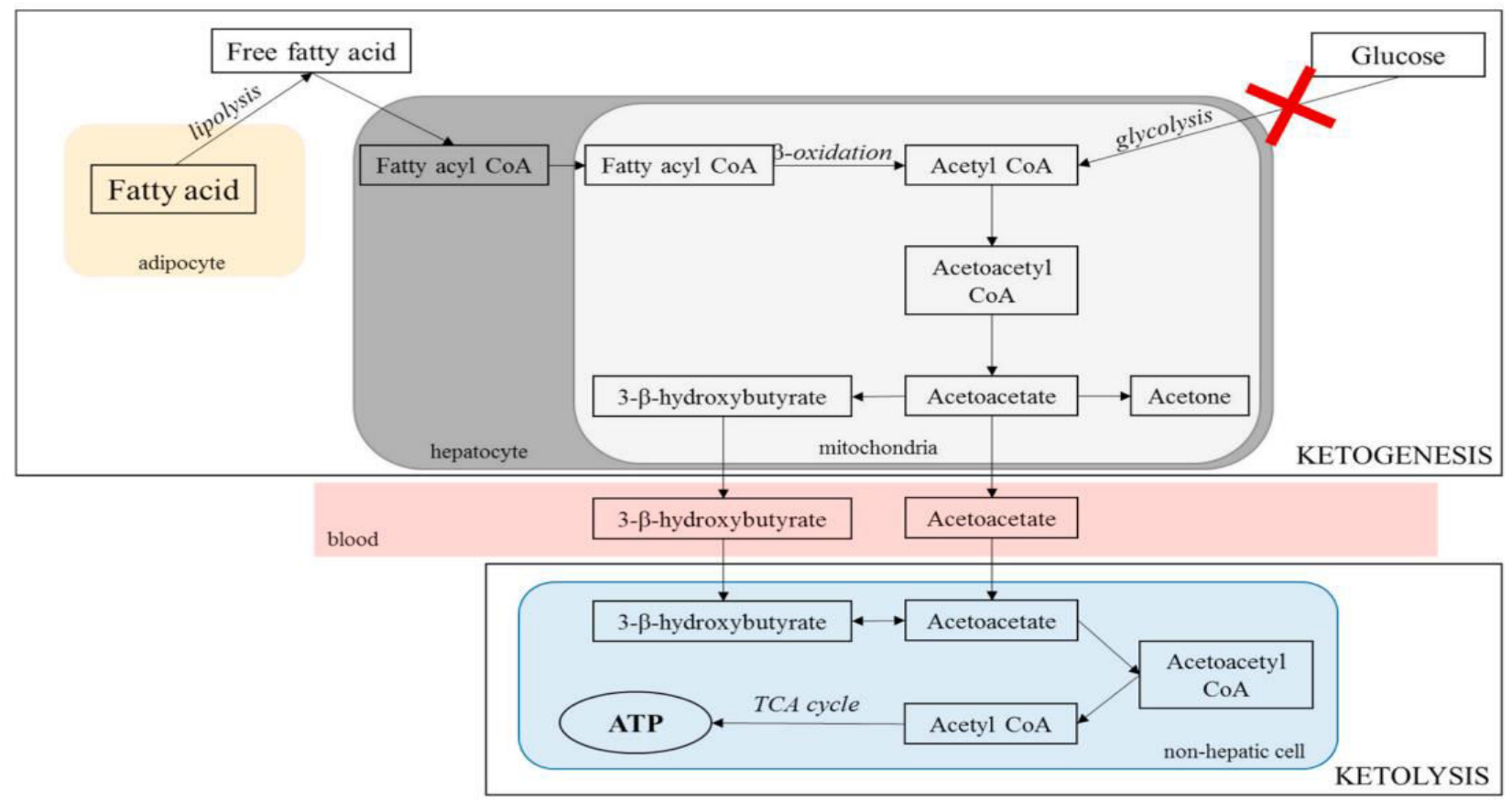

Fig. 3: Simplified pathways of ketone metabolism during KD (Drabińska et al., 2021).

KD was found to have positive effects on body weight, BMI, waist circumference, HDL levels, triglyceride levels, blood sugar level, hemoglobin A1c (HbA1c), and insulin levels. However, there was not enough record to support ketogenic diet for long time because most of the studies were of shorter duration, i.e., ranging from 3 months to 3 years. Although most studies mentioned the shift in lipid profile to be favorable, increases were also found in TC and LDL (Batch et al., 2020). Some studies showed that significant differences were found in the first 6 months of intervention, but this statistical significance decreased after one to two years. Meager sample sizes, brief study durations, and absence of control diets decrease validity of these studies (Drabińska et al., 2021). Majority of the studies in the present review had fewer than 50 participants, were of about 3 months duration, and about half of the studies did not have controls. As there is restrained number of strong studies depicting the potential risks of $\mathrm{KD}$, recommendations to support ketogenic diet in patients without comorbidities, should be done by the nutritionist (Batch et al., 2020). VLCKD is a great therapy for people with obesity and especially for those who have already been unsuccessful in losing weight in the past, and/or have an urgency to lose weight. Once the target weight is achieved, it is compulsory to suggest an appropriate healthy lifestyle i.e.

UniversePG I www.universepg.com physical activity and a balanced nutritional pattern (Drabińska et al., 2021).

\section{CONCLUSION:}

Our literature review found considerable favorable effects of both KD and VLCKD on fat mass and lipid profile, though the studies included in the review included 12 months dietary intervention only. More studies need to be done for extended periods of time (at least $\geq 2$ years) to observe effects of these diets after long duration.

\section{ACKNOWLEDGEMENT:}

We thank our colleagues for their moral support and guidance.

\section{CONFLICTS OF INTEREST:}

There is no conflict of interest.

\section{REFERENCES:}

1) Albanese, A., Vettor, R., Foletto, M., (2019). Pre-operative Very Low Calorie Ketogenic Diet (VLCKD) vs. Very Low Calorie Diet (VLCD): Surgical Impact. OBES SURG 29, 292-296. https://doi.org/10.1007/s11695-018-3523-2

2) Batch, J.T., Sultan, S., Ramirez, M.N., (2020). Advantages and Disadvantages of the Ketogenic Diet: A Review Article. Cureus. https://doi.org/10.7759/cureus.9639 
3) Blanco, J. C., Khatri, A., Kifayat, A., Cho, R., Aronow, W.S., (2019). Starvation Ketoacidosis due to the Ketogenic Diet and Prolonged Fasting - A Possibly Dangerous Diet Trend. Am J Case Rep, 20, 1728-1731.

https://doi.org/10.12659/AJCR.917226

4) Bruci, A., Tozzi, R., Balena, A., Watanabe, M., (2020). Very Low-Calorie Ketogenic Diet: A Safe and Effective Tool for Weight Loss in Patients with Obesity and Mild Kidney Failure. Nutrients, 12, 333.

https://doi.org/10.3390/nu12020333

5) Buscemi, S., Buscemi, C., Ciaccio, M., (2021). Obesity and Circulating Levels of Vita-min D before and after Weight Loss Induced by a Very Low-Calorie Ketogenic Diet. Nutrients, 13, 1829. https://doi.org/10.3390/nu13061829

6) Castellana, M., Conte, E., Trimboli, P., (2020). Efficacy and safety of very low calorie ketogenic diet (VLCKD) in patients with overweight and obesity: A systematic re-view and meta-analysis. Rev Endocr Metab Disord, 21, 5-16. https://doi.org/10.1007/s11154-019-09514-y

7) Castro, A., Aguera, Z., Casanueva, F., (2018). Effect of A Very Low-Calorie Ketogenic Diet on Food and Alcohol Cravings, Physical and Sexual Activity, Sleep Disturbances, and Quality of Life in Obese Patients. Nutrients, 10, 1348.

https://doi.org/10.3390/nu10101348

8) Choi, H.-R., Kim, J., Lim, H., Park, Y., (2018). Two-Week Exclusive Supplementation of Modified Ketogenic Nutrition Drink Reserves Lean Body Mass and Improves Blood Lipid Profile in Obese Adults: A Randomized Clinical Trial. Nutrients, 10, 1895.

https://doi.org/10.3390/nu10121895

9) D’Abbondanza, M., Pucci, G., Vaudo, G., (2020). Very Low-Carbohydrate Ketogenic Diet for the Treatment of Severe Obesity and Associated Non- Alcoholic Fatty Liver Disease: The Role of Sex Differences. Nutrients, 12, 2748.

https://doi.org/10.3390/nu12092748

10) Di Raimondo, D., Corleo, D., Pinto, A., (2021). Ketogenic Diet, Phy-sical Activity, and Hypertension - A Narrative Review. Nutrients, 13, 2567. https://doi.org/10.3390/nu13082567
11) Drabińska, N., Wiczkowski, W., Piskuła, M.K., (2021). Recent advances in the application of a ketogenic diet for obesity management. Trends in Food Science \& Technology, 110, 28-38. https://doi.org/10.1016/j.tifs.2021.01.080

12) Gomez-Arbelaez, D., Castro, A.I., Casanueva, F.F., (2018). Resting metabolic rate of obese patients under very low calorie ketogenic diet. NutrMetab (Lond), 15, 18. https://doi.org/10.1186/s12986-018-0249-Z

13) Greene, D.A., Chap-man, P., Rigney, M., (2018). A Low-Carbohydrate Ketogenic Diet Reduces Body Mass Without Compromising Performance in Power-lifting and Olympic Weightlifting Athletes. Journal of Strength and Conditioning Research, 32, 3373-3382. https://doi.org/10.1519/JSC.0000000000002904

14) Gupta, L., Kalra, S., Gupta, P., Dutta, D., (2017). Ketogenic diet in endocrine disorders: Current perspectives. J Postgrad Med, 63 (242). https://doi.org/10.4103/jpgm.JPGM_16_17

15) Hall, K.D., Guo, J., Chung, S.T., (2020). A plantbased, low-fat diet decreases ad libitum energy intake compared to an animal-based, ketogenic diet: An inpatient randomized cont-rolled trial (preprint). NutriXiv.

https://doi.org/10.31232/osf.io/rdjfb

16) Luukkonen, P.K., Lyu, K., Yki-Järv-inen, H., (2020). Effect of a ketogenic diet on hepatic steatosis and hepatic mitochondrial metabolism in nonalcoholic fatty liver disease. Proc Natl Acad Sci USA, 117, 7347-7354. https://doi.org/10.1073/pnas.1922344117

17) McKenzie, A.L., Volk, B.M., Link, T.M., (2017). A Novel Intervention Including Indiv-idualized Nutritional Recommendations Reduces Hemoglobin A1c Level, Medication Use, and Weight in Type 2 Diabetes. JMIR Diabetes, 2 , e5. https://doi.org/10.2196/diabetes.6981

18) Merra, G., Gratteri, S., Renzo, L.D., (2017). Effects of very-low-calorie diet on body composition, Metabolic state, and genes expression, 17.

19) Ministrini, S., Calzini, L., Lupattelli, G., (2019). Lysosomal Acid Lipase as a Molecular Target of the Very Low Carbo-hydrate Ketogenic Diet in Morbidly Obese Pati-ents: The Potential Effects 
on Liver Steatosis \& Cardiovascular Risk Factors. JCM, 8(621). https://doi.org/10.3390/jcm8050621

20) Muscogiuri, G., Barrea, L., Colao, A., (2019). The management of very low-calorie ketogenic diet in obesity outpatient clinic: a practical guide. J Transl Med, 17(356).

https://doi.org/10.1186/s12967-019-2104-z

21) Myette-Côté, É., Durrer, C., Little, J.P., (2018). The effect of a short-term low-carbohydrate, high-fat diet with or without postmeal walks on glycemic control and inflammation in type 2 diabetes: a rand-omized trial. American Journal of Physiology-Regulatory, Integrative and Comparative Physiology, 315, R1210-R1219. https://doi.org/10.1152/ajpregu.00240.2018

22) Perticone, M., Maio, R., Perticone, F., (2019). Ketogenic Diet-Induced Weight Loss is Associated with an Increase in Vitamin D Levels in Obese Adults. Molecules, 24(2499). https://doi.org/10.3390/molecules24132499

23) Pilone, V., Berselli, T., Schiavo, L., (2018). Metabolic effects, safety, and acce-ptability of very low-calorie ketogenic dietetic scheme on candidates for bariatric surgery. Surgery for Obesity and Related Diseases, 14, 1013-1019. https://doi.org/10.1016/j.soard.2018.03.018

24) Sajoux, Lorenzo, Martinez, Casanueva, (2019). Effect of a Very-Low-Calorie Ketogenic Diet on Circulating Myokine Levels Compared with the Effect of Bariatric Surgery or a Low-Calorie Diet in Patients with Obesity. Nutrients, 11(2368). https://doi.org/10.3390/nu11102368

25) Saslow, L. R., Moran, P., Hecht, F. M., (2017). Twelve-month outcomes of a randomized trial of a moderate-carbohydrate versus very low-carbohydrate diet in overweight adults with type2 DM or prediabetes. Nutr \& Diabetes, 7(304). https://doi.org/10.1038/s41387-017-0006-9
26) Schiavo, L., Pilone, V., Iannelli, A., (2018). A 4Week Preoperative Ketogenic MicronutrientEnriched Diet Is Effective in Reducing Body Weight, Left Hepatic Lobe Volume, and Micronutrient Deficiencies in Patients Undergoing Bariatric Surgery: a Prospective Pilot Study. OBES SURG 28, 2215-2224. https://doi.org/10.1007/s11695-018-3145-8

27) Valenzano, A., Polito, R., Monda, V., (2019). Effects of Very Low Calorie Ketogenic Diet on the Orexinergic System, Visceral Adipose Tissue, and ROS Pro-duction. Antioxidants, 8(643). https://doi.org/10.3390/antiox8120643

28) Volek, J.S., LaFountain, R.A., Dituro, P., (2019). Extended Ketogenic Diet and Physical Training Intervention in Military Personnel. Military Medicine, 184, 199-200. https://doi.org/10.1093/milmed/usz184

29) Walton, C.M., Perry, K., Hart, R.H., (2019). Improvement in Glycemic and Lipid Profiles in Type 2 Diabetics with a 90-Day Ketogenic Diet. Journal of Diabetes Research 2019, 1-6. https://doi.org/10.1155/2019/8681959

30) Watanabe, M., Tozzi, R., Risi, R., (2020). Beneficial effects of the ketogenic diet on non-alcoholic fatty liver disease: A comprehensive review of the literature. Obesity Reviews, 21. https://doi.org/10.1111/obr.13024

31) Yuan, X., Wang, J., Sun, C., (2020). Eff-ect of the ketogenic diet on glycemic control, insulin resistance, and lipid metabolism in pati-ents with T2DM: a systematic review and meta-analysis. Nutr. Diabetes, 10(38). https://doi.org/10.1038/s41387-020-00142-z

Citation: Kanwal N, Kashif S, Kashif M, Allaudin, and Rehman G. (2022). Effect of a ketogenic diet on body weight and lipid profile. Eur. J. Med. Health Sci., 4(1), 08-17. https://doi.org/10.34104/ejmhs.022.08017 @) () 